\title{
Robot-aided thoracoscopic thymectomy for early-stage thymoma: A multicenter European study
}

\author{
Giuseppe Marulli, MD, PhD, ${ }^{\mathrm{a}}$ Federico Rea, MD, ${ }^{\mathrm{a}}$ Franca Melfi, MD, ${ }^{\mathrm{b}}$ Thomas A. Schmid, MD, ${ }^{\mathrm{c}}$ \\ Mahmoud Ismail, MD, ${ }^{\mathrm{d}}$ Olivia Fanucchi, MD,${ }^{\mathrm{b}}$ Florian Augustin, MD, ${ }^{\mathrm{c}}$ Marc Swierzy, MD,${ }^{\mathrm{d}}$ \\ Francesco Di Chiara, MD, ${ }^{\mathrm{a}}$ Alfredo Mussi, MD, ${ }^{\mathrm{b}}$ and Jens C. Rueckert, $\mathrm{MD}^{\mathrm{d}}$
}

\begin{abstract}
Objective: Minimally invasive thymectomy for stage I to stage II thymoma has been suggested in recent years and considered technically feasible. However, because of the lack of data on long-term results, controversies still exist on surgical access indication. We sought to evaluate the results after robot-assisted thoracoscopic thymectomy in early-stage thymoma.
\end{abstract}

\begin{abstract}
Methods: Data were collected from 4 European centers. Between 2002 and 2011, 79 patients (38 men and 41 women; median age, 57 years) with early-stage thymoma were operated by left-sided $(82.4 \%)$, rightsided $(12.6 \%)$, or bilateral $(5 \%)$ robotic thoracoscopic approach. Forty-five patients $(57 \%)$ had associated myasthenia gravis.
\end{abstract}

\begin{abstract}
Results: Average operative time was 155 minutes (range, 70-320 minutes). One patient needed open conversion, in 1 patient a standard thoracoscopy was performed after robotic system breakdown, and in 5 patients an additional access was required. No vascular and nervous injuries were recorded, and no perioperative mortality occurred. Ten patients $(12.7 \%$ ) had postoperative complications. Median hospital stay was 3 days (range, 2-15 days). Median diameter of tumor resected was $3 \mathrm{~cm}$ (range, 1-12 cm), and Masaoka stage was stage I in 30 patients $(38 \%)$ and stage II in 49 patients $(62 \%)$. At a median follow-up of 40 months, 74 patients were alive and 5 had died (4 patients from nonthymoma-related causes and 1 from a diffuse intrathoracic recurrence), with a 5 -year survival rate of $90 \%$.
\end{abstract}

Conclusions: Our data indicate that robot-enhanced thoracoscopic thymectomy for early-stage thymoma is a technically sound and safe procedure with a low complication rate and a short hospital stay. Oncologic outcome seems good, but a longer follow-up is needed to consider this as a standard approach definitively. (J Thorac Cardiovasc Surg 2012;144:1125-32)

Thymoma is the most common primary tumor in the anterior mediastinum of adults. A complete surgical resection with safety margins is considered curative for resectable tumors and represents the most important prognostic factor. ${ }^{1}$ During the past 2 decades, video-assisted thoracoscopic surgery (VATS) has been performed frequently for the treatment of mediastinal benign diseases or for thymectomy in cases of nonthymomatous myasthenia gravis (MG). ${ }^{2,3}$ However, in patients with thymoma, a total thymectomy

\footnotetext{
From the Thoracic Surgery Division, ${ }^{\text {a }}$ Department of Cardiac, Thoracic and Vascular Sciences, University of Padova, Padova, Italy; Thoracic Surgery Division, ${ }^{\mathrm{b}}$ Department of Cardiac, Thoracic and Vascular Surgery, University of Pisa, Pisa, Italy; Department of Visceral, Transplant, and Thoracic Surgery, ${ }^{\mathrm{c}}$ Innsbruck Medical University, Innsbruck, Austria; and Thoracic Surgery Division, ${ }^{\mathrm{d}}$ Charité Campus Mitte, Universitätsmedizin Berlin, Berlin, Germany.

Disclosures: Authors have nothing to disclose with regard to commercial support.

Read at the 92nd Annual Meeting of The American Association for Thoracic Surgery, San Francisco, California, April 28-May 2, 2012.

Received for publication April 25, 2012; revisions received July 10, 2012; accepted for publication July 30, 2012; available ahead of print Sept 3, 2012.

Address for reprints: Giuseppe Marulli, MD, PhD, Department of Cardiologic, Thoracic and Vascular Sciences, Division of Thoracic Surgery, University of Padova, Via Giustiniani, 2 35100, Padova, Italy (E-mail: giuseppe.marulli@ unipd.it).

$0022-5223 / \$ 36.00$

Copyright (C) 2012 by The American Association for Thoracic Surgery

http://dx.doi.org/10.1016/j.jtcvs.2012.07.082
}

by a median sternotomy has been universally accepted as the standard treatment. ${ }^{4}$ The VATS approach for thymoma remains controversial, and many surgeons are reluctant to use this technique because of the supposed increased risk of local recurrence, reduced safety margins after minimally invasive resection, possible rupture of the capsule, and seeding of the tumor during endoscopic manipulations. Furthermore, the lack of long-term data on survival and oncologic results, and the difficult learning curve for performing the operation are additional reasons to support any doubts regarding the effectiveness of VATS resection for earlystage thymomas. ${ }^{5}$ The introduction of robotic-assisted technologies during the late 1990s provided a technical improvement that overcame the limitations of conventional thoracoscopy. In fact, the 3-dimensional vision system and the multiarticulated instruments of the da Vinci Surgical Robotic System (Intuitive Surgical, Inc, Sunnyvale, Calif) have added further precision and dexterity. This technology has been tested in a variety of thoracic surgery procedures, with clear evidence that the robotic system provides its best advantage when operating in tiny and difficult-to-reach anatomic regions such as the mediastinum. Therefore, mediastinal diseases were selected electively by most surgeons for clinical research using the da Vinci robot. ${ }^{6,7}$ The aim of this 


\section{Abbreviations and Acronyms \\ MG = myasthenia gravis \\ VATS $=$ video-assisted thoracoscopic surgery}

study was to evaluate the safety and feasibility of robotic thymectomy by analyzing the oncologic outcomes in a group of patients affected by early-stage thymoma and operated on in 4 European Centers with significant experience in this kind of operation.

\section{MATERIALS AND METHODS}

We reviewed the data of 79 patients undergoing robotic thymectomy for clinically defined early-stage thymoma (Masaoka stages I and II) collected between February 2002 and March 2011 by 4 European Thoracic Surgery Centers (University of Berlin, Germany, 39 patients; University of Padova, Italy, 14 patients; University of Pisa, Italy, 13 patients; University of Innsbruck, Austria, 13 patients). All patients signed a detailed consent form in which they were informed about possible complications of thymoma resection by the robotic thoracoscopic approach and the lack of long-term data. The institutional review board of each center approved this study. Information on patient demographics, presence of associated MG, tumor characteristics, stage, intra- and postoperative data (eg, complications, need for open conversion or additional ports or accesses, operative time, length of hospital stay), and administration of adjuvant treatment were collected. The Masaoka staging system was used to assess the pathological stage, ${ }^{8}$ whereas the new World Health Organization classification was used for defining the histology. ${ }^{9}$ The Myasthenia Gravis Foundation of America classification ${ }^{10}$ was applied to stratify the preoperative class of MG. Preoperative assessments included an evaluation of pulmonary and cardiac function, and total body computed tomography or magnetic resonance imaging. Preferred radiologic characteristics to be eligible for robotic thymectomy were as follows: location of the tumor in the anterior mediastinum; a distinct fat plane between the tumor and surrounding structures; unilateral tumor predominance; tumor encapsulation; existence of residual, normalappearing thymic tissue; and no mass compression effect (Figure 1). ${ }^{11}$ Tumor size was not considered a strict selection criterion; however, a diameter $\leq 5 \mathrm{~cm}$ was preferred; an evident unilateral predominance was essential to start the robotic procedure for tumors $>5 \mathrm{~cm}$. Exclusion criteria were radiologic evidence of invasion of surrounding structures (eg, pericardium, lung, nerves, or large vessels), the presence of adhesions (eg, previous thoracic surgery or pleuritis), the inability to perform single-lung ventilation, or a body mass index $>35$. All patients completed the follow-up (the last follow-up was March 2012) with a periodic clinical evaluation. Chest and abdominal computed tomographic scans were required every 6 months during the first 2 years after surgery, and then every year. Patients with MG were also evaluated periodically by the referring neurologists and were sent for surgical evaluation in case of worsening MG symptoms.

\section{Surgical Technique}

A 3- or 4-arm da Vinci robotic system was used. Briefly, the general setup was as follows. The patient was positioned left side up at a $30^{\circ}$ angle using a bean bag. A camera port for the 3 -dimensional $0^{\circ}$ stereo-endoscope was introduced through a $15-\mathrm{mm}$ incision in the fifth intercostal space on the midaxillary line. Care was taken when operating on the left side to avoid the heart, which lies just beneath this area. Thus, under camera vision and carbon dioxide insufflation to turn away the heart, 2 additional thoracic ports were inserted: one in the third intercostal space on the midaxillary line and another in the fifth intercostal space between the parasternal and the midclavicular lines (Figure 2). The robotic thymectomy surgical technique either from the left or right side has been described previously. ${ }^{12,13}$ We emphasize some technical tips and pitfalls. Unlike the thoracoscopic approach for a nonthymomatous gland, some oncologic modifications were adopted in the case of a thymoma resection. All surgeons involved adopted a "no-touch technique" with an en-bloc resection of the thymus and perithymic fat tissue. During this technique, the thymoma was never touched, and normal thymic tissue and perithymic fat were used for grasping and for traction, to avoid direct manipulation of the tumor, capsular damage, and potential seeding. All thymic and perithymic fat was dissected with safe surgical margins, according to the International Thymic Malignancy Interest Group criteria, ${ }^{14}$ and the completeness of the thymectomy was assessed by macroscopic inspection of the thymic bed and the specimen (Figure 3). Either a spatula, hook monopolar coagulators, or an ultrasonic dissector were used routinely for dissection, focusing on avoiding thermal injury particularly when operating close to the phrenic nerves. Carbon dioxide insufflation of 8 to $12 \mathrm{~mm} \mathrm{Hg}$ was applied during the procedure to increase the size of the mediastinal space.

\section{Statistical Analysis}

Data are expressed as median or mean \pm standard deviation. The normality of the quantitative variable distribution was verified by the Shapiro-Wilk test. The Student $t$ test for paired data or Wilcoxon signedrank test was used, as appropriate, to evaluate differences between means. Survival curves were calculated by the Kaplan-Meier method. A $P$ value $<.05$ was considered to indicate statistical significance.

\section{RESULTS}

Thirty-eight men $(48.1 \%)$ and 41 women $(51.9 \%)$ were enrolled, with a median age of 57 years (range, 13-84 years). Forty-five patients $(56.9 \%$ ) were affected by MG. Preoperative Myasthenia Gravis Foundation of America class was I in 9 patients, IIA in 4 patients, IIB in 11 patients, IIIA in 11 patients, IIIB in 7 patients, IVA in 2 patients, and IVB in 1 patient. The robotic thoracoscopic surgical approach was left-sided in 65 patients $(82.3 \%)$, right-sided in 10 patients $(12.6 \%)$, and bilateral in 4 patients $(5.1 \%)$. Median operative time was 155 minutes, (range, 70-320 minutes; mean, $165 \pm 52$ minutes). One patient (1.3\%) required conversion to an open approach because of a largediameter tumor interfering with a safe dissection. One patients $(1.3 \%)$ required a standard thoracoscopy after the robotic system broke down. Five patients $(6.3 \%)$ needed additional access (cervicotomy in 1 patients as a result of a difficult dissection plane in the neck independent from the tumor, and an additional omolateral thoracoscopic port for suction purposes in 4 patients). No vascular or nerve injuries were recorded and no perioperative mortality occurred. Ten patients $(12.7 \%)$ had postoperative complications: 1 pneumothorax treated with a chest tube, 1 hemothorax treated conservatively with a blood transfusion, 3 pleural effusions, 1 case of pneumonia, 1 case of orthostatic hypotension, 1 atrial fibrillation, 1 wound infection, and 1 urinary tract infection treated with medical therapy. Median hospital stay was 3 days (range, 2-15 days; mean, $4.4 \pm 2.5$ days). Median time to chest drainage removal was 2 days (range, $1-8$ days; mean, $2.4 \pm 1.6$ days). Median diameter of the resected tumors was $3 \mathrm{~cm}$ (range, 1-12 cm; 


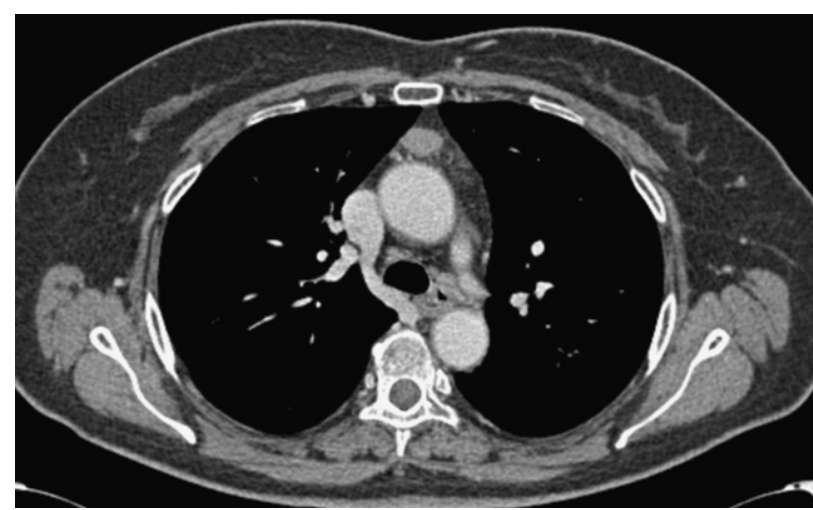

FIGURE 1. Computed tomographic scan of an early-stage thymoma.

mean, $3.7 \pm 2 \mathrm{~cm}$ ). Masaoka stage was I in 30 patients $(38 \%)$ and II in 49 patients $(62 \%)$. World Health Organization histology was type $\mathrm{A}$ in 14 patients $(17.7 \%), \mathrm{AB}$ in 22 patients $(27.8 \%), \mathrm{B} 1$ in 12 patients $(15.2 \%), \mathrm{B} 2$ in 18 patients $(22.8 \%), \mathrm{B} 3$ in 12 patients $(15.2 \%)$ and $\mathrm{C}$ in 1 patient $(1.3 \%)$. No relationship was found between tumor diameter and Masaoka stage (stage I mean diameter, $3.6 \pm 2.1 \mathrm{~cm}$; stage II mean diameter, $3.7 \pm 1.9 \mathrm{~cm} ; P=.88$ ), and no significant difference in operative time was seen when operating on tumors with dimensions $>3 \mathrm{~cm}$ (mean operative time for tumors $\leq 3 \mathrm{~cm} 155 \pm 49$ minutes vs $175 \pm 53$ minutes for tumors $>3 \mathrm{~cm} ; P=.10$ ). Postoperative radiation therapy was administered in 18 cases, all with stage II disease. At the last follow-up (median, 40 months; range, 12-121 months; mean, $51.7 \pm 33$ months), 74 patients were alive, 4 patients had died of nonthymoma-related causes (leukemia, vulvar carcinoma, pneumonia, and sepsis), and 1 patient (type $\mathrm{C}, 5-\mathrm{cm}$ tumor) died because of a diffuse intrathoracic recurrence. The 5-year overall survival rate was $90 \%$ (Figure $4, A$ ); the 5-year thymoma-related survival was $97 \%$ (Figure $4, B$ ).

\section{DISCUSSION}

In a systematic review, Davenport and colleagues ${ }^{15}$ reported an overall 5-year survival rate for patients with Masaoka stage I and stage II thymoma, after complete surgical resection, ranging from $89 \%$ to $100 \%$ and $71 \%$ to $95 \%$, respectively. The authors pointed out the lack of evidence in the current literature supporting a minimally invasive approach compared with a standard transsternal approach. In particular, few authors have reviewed systematically the surgical results of thoracoscopic (conventional or robot-assisted) thymectomy for early-stage thymoma (Table $1^{1,2,11,16-20}$ ). The available data were sufficient to confirm only that this approach could be considered technically sound and safe in the hands of appropriately trained surgeons. However, data were inconclusive with regard to oncologic outcome, which was primarily the result of the long (10-year) lapse of time necessary in patients with thymoma to evaluate the survival and relapse rate. At that time, the open transsternal surgical approach was widely considered the gold standard for resecting

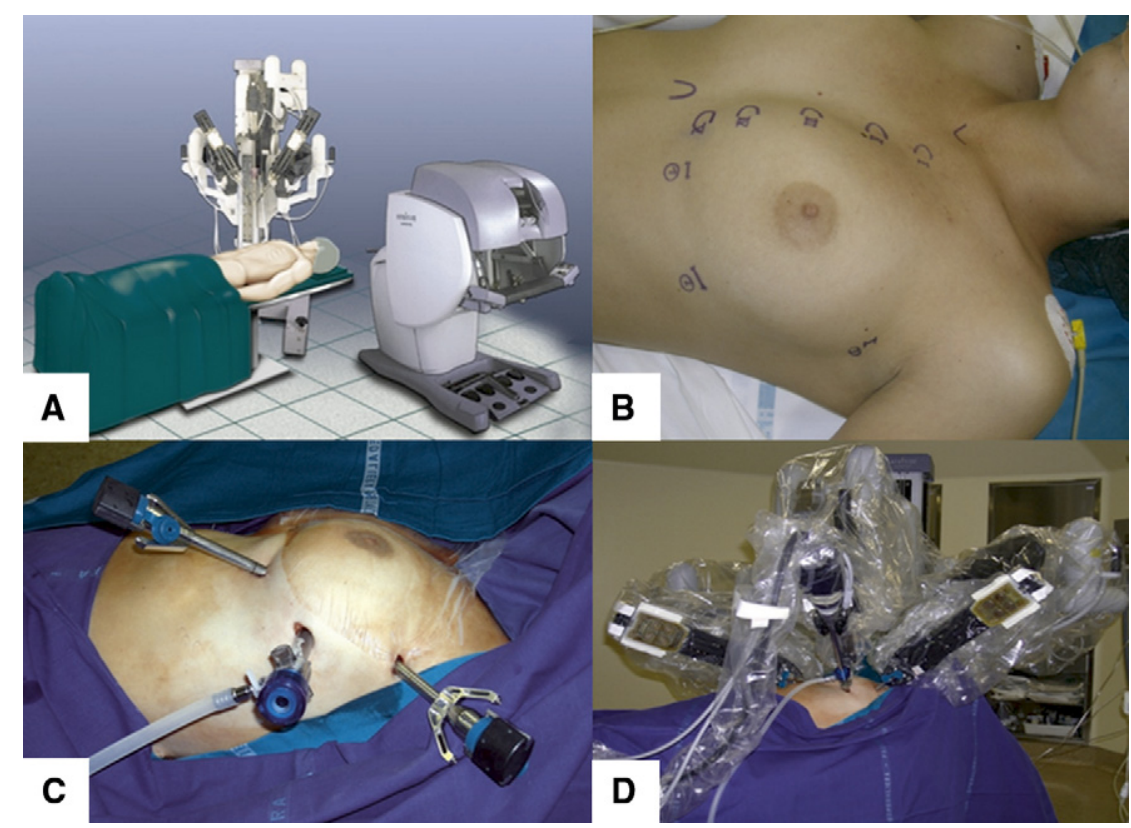

FIGURE 2. A, In the surgical room, the patient is positioned supine on the surgical table with the left side elevated at $30^{\circ}$. The left arm is flexed on a support to expose the axillary region. The da Vinci robotic system with the surgical cart and the surgeon's console are displaced. B, Before surgery the fifth and third intercostal spaces (sites of incisions for port placement) are identified. The thoracic ports are placed, $(\mathrm{C})$ and the arms of the robot are then attached to the ports and are operative (D). Conflict between arms should be avoided. 


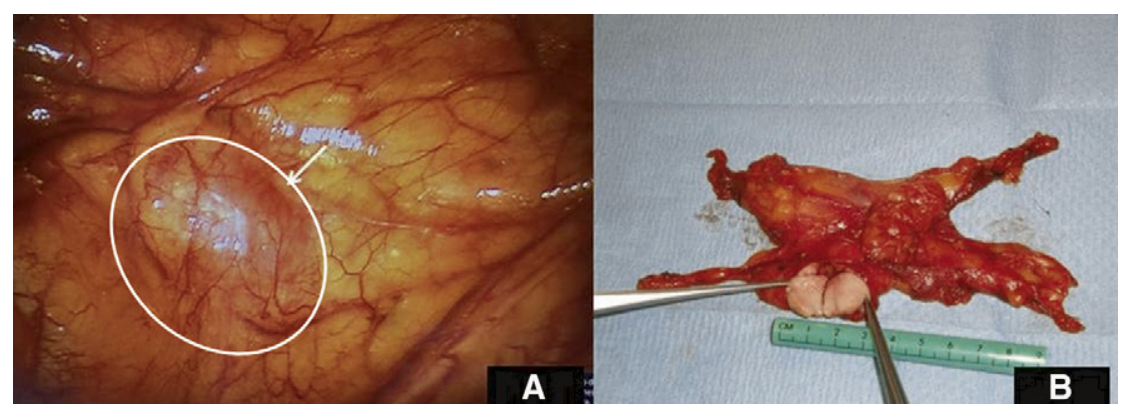

FIGURE 3. A, Intraoperative left thoracoscopic view of an encapsulated thymoma (white arrow). B, Specimen after thymectomy.

a thymoma and it ensured the best chances of a complete resection. ${ }^{1,4}$ VATS was introduced in clinical practice during the early 1990s and soon gained broad acceptance for diagnostic and therapeutic interventions for benign mediastinal diseases. ${ }^{2,3}$ The advantages of this minimally invasive approach have resulted in greater acceptability, particularly among patients with $\mathrm{MG}$, leading to an increased number of thoracoscopic thymectomies being performed for nonthymomatous MG with good surgical and neurologic results. ${ }^{12,13}$ However, most surgeons are still reluctant to undertake a thoracoscopic-assisted thymectomy in patients with thymoma because of technical and oncologic concerns.

\section{Technical Aspects}

The upper mediastinum is a delicate and difficult-toreach anatomic area for thoracoscopy, with large, vulnerable vessels and nerves. The 2-dimensional view of the operative field, the surgeon's tremor enhanced by the thoracoscopic instruments, and the fact that the instruments do not articulate make it difficult to operate in a fixed, tiny 3-dimensional space such as the mediastinum. Moreover, thoracoscopic thymectomy is considered a technically challenging operation that requires a long learning curve. ${ }^{5}$ The introduction of robotic surgical systems added a new dimension to conventional thoracoscopy, providing additional advantages and overcoming some technical and methodological limits. First, the improved dexterity of instruments that can articulate with $7^{\circ}$ of freedom and rotate $360^{\circ}$ allows complex 3-dimensional movements superior to those permitted by conventional minimally invasive instruments, enhances dissection safety around vessels and nerves, and is more comfortable in tiny, remote areas such as the superior horns or the contralateral mediastinum. Second, the high-resolution, 3-dimensional real-time video image permits the best possible and magnified view of the surgical field. Third, the filtering of hand tremors allows greater technical precision.

In our series, our preference was for a left-sided approach for technical, clinical, and anatomic reasons. As demonstrated by Rückert and associates, ${ }^{21}$ the larger part of the thymus is on the left side. Furthermore, in about $72 \%$ of patients $^{22}$ the thymus gland is located lateral and under the left phrenic nerve, which is better identified and dissected safely under direct vision from the left. The right phrenic nerve is protected partially by the superior vena cava in the high mediastinum and may be identified and followed easily in the lower part. For patients with MG, all mediastinal fat tissue was removed, comprising an aortopulmonary window, which is a frequent side of ectopic thymic tissue, and it is easier to reach by the left-sided approach. The right-sided
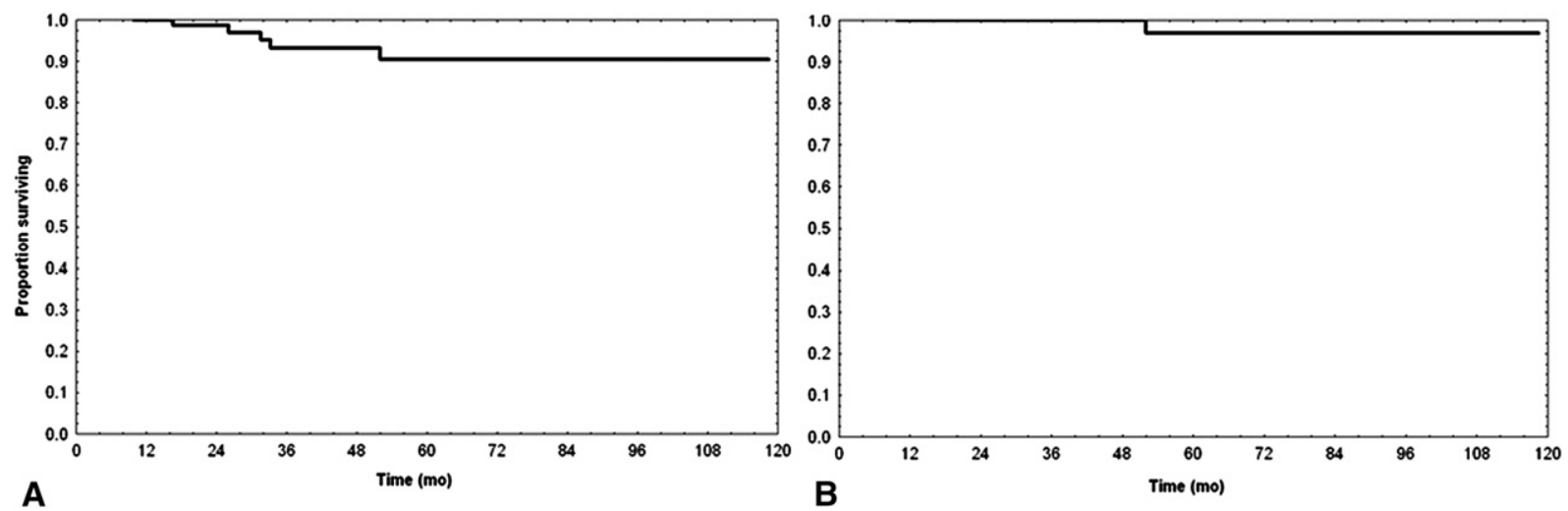

FIGURE 4. A, Overall survival. B, Thymoma-related survival. 
TABLE 1. Review of the published studies on thoracoscopic thymectomy for early-stage thymoma

\begin{tabular}{|c|c|c|c|c|c|c|c|c|c|c|}
\hline Authors & $\begin{array}{c}\text { No. of } \\
\text { patients }\end{array}$ & SA & $\begin{array}{l}\text { Masaoka } \\
\text { stage I/II }\end{array}$ & $\mathrm{TS}, \mathrm{cm}$ & $\begin{array}{c}5 \text {-Year } \\
\text { survival, \% }\end{array}$ & Follow-up, mo & $\mathbf{R R}, \%$ & OC, $\%$ & $\begin{array}{c}\text { OT, } \\
\text { minutes }\end{array}$ & $\begin{array}{l}\text { POS, } \\
\text { days }\end{array}$ \\
\hline Roviaro and colleagues $^{2}$ & 22 & uVATS & 22 & - & - & - & 4.5 & 4.5 & $75^{*}$ & $6^{*}$ \\
\hline Cheng and colleagues ${ }^{11}$ & 44 & uVATS & $27 / 17$ & $7.7 *$ & 100 & $34.6^{*}$ & 0 & 0 & $194 *$ & $7.6^{*}$ \\
\hline Odaka and colleagues ${ }^{16}$ & 22 & uVATS & - & - & - & $21.6^{*}$ & 0 & 0 & $194^{*}$ & $4.6^{*}$ \\
\hline Agasthian and $\operatorname{Lin}^{17}$ & 50 & uVATS & $25 / 25$ & $5^{*}$ & 100 & $58 *$ & 2 & 0 & $150^{*}$ & $5^{*}$ \\
\hline Pennathur and colleagues ${ }^{18}$ & 18 & bVATS & $5 / 13$ & $3.5^{*}$ & 100 & $27 \dagger$ & 0 & 0 & - & $2.9^{*}$ \\
\hline Takeo and colleagues $^{19}$ & 34 & bVATS & $15 / 19$ & $5.2^{*}$ & 100 & $65^{*}$ & 2.8 & 0 & $219^{*}$ & $10.5^{*}$ \\
\hline Mussi and colleagues ${ }^{20}$ & 13 & Robotic & $7 / 6$ & $3.3^{*}$ & 100 & $14.5 \dagger$ & 0 & 7.7 & $139 *$ & $4^{*}$ \\
\hline Current series & 79 & Robotic & $30 / 49$ & $3.7^{*}$ & 90 & $51.7 *$ & 1.3 & 1.3 & $165^{*}$ & $4.4^{*}$ \\
\hline
\end{tabular}

$S A$, Surgical access; $T S$, tumor size; $R R$, recurrence rate; $O C$, open conversion; $O T$, operative time; $P O S$, postoperative length of stay; $u V A T S$, unilateral video-assisted thoracic surgery; $b$ VATS, bilateral video-assisted thoracic surgery. *Mean value. $\dagger$ Median value.

approach was reserved mainly for tumors located on the right side.

\section{Safety}

The main oncologic concern is related to possible breach of the tumor capsule, with risk of tumor seeding locally or in the pleural cavity, and the difficult evaluation of resection margins with reduced oncologic accuracy and safety. In our opinion, the robotic approach has some clear advantages compared with conventional thoracoscopy. In fact, a lesser manipulation of the thymic and perithymic tissue is required during the operation, and a better evaluation of healthy tissue as a result of the high-quality images leads to a more precise and low-risk dissection, with wide safety margins and reduced possibility of tumor breaching, incomplete resection, or iatrogenic injury. The use of carbon dioxide inflation (usually $8-12 \mathrm{~mm} \mathrm{Hg}$ ) during the operation is another advantage, which allows enlargement of the mediastinal space with better visualization. The lack of tactile feedback could, theoretically, increase the risk of damaging tumor tissue; however, this disadvantage seems compensated for by the superior 3-dimensional vision control of the system. No complications based on a lack of tactile feedback were observed in our series. The completeness of the procedure was proved by intraoperative evaluation of the resection margins, back-table investigation of the specimens, and the histological analysis.

\section{Surgical and Oncologic Results}

Different from other authors, ${ }^{16,23}$ we support a thoracoscopic subtotal thymectomy for noninvasive thymoma without MG as the preferred resection modality, regardless of tumor size or the nature of its surrounding capsule. Our policy was to undertake an extended thymectomy in all cases, such as in an open approach. In the absence of definitive long-term data standardization of the technique, it is necessary to avoid biases during outcome evaluations. Moreover, we consider the intraoperative manipulation of the specimen safer when the perithymic fat tissue is contextually resected en bloc. A further debated point is the appropriate size of thymoma for VATS or robotic resection. The majority of the literature considers lesions $\leq 5$ $\mathrm{cm}$, but an average-size tumor of $3 \mathrm{~cm}$ is generally considered acceptable oncologically. ${ }^{5}$ In our experience, the median diameter of resected lesions was $3 \mathrm{~cm}$, with a range of 1 to $12 \mathrm{~cm}$. Large tumor size was not considered an absolute contraindication; however, it may interfere with the thoracoscopic procedure, because the manipulation is more difficult, with an increased chance of an open conversion, or it may prolong operative time. In fact, the single case requiring an open conversion in our series was a 6-cm-diameter tumor in which the manipulation was considered a risk. As a general rule, we follow the radiologic criteria proposed by Cheng and coworkers ${ }^{11}$ to select candidates for thoracoscopic thymectomy in patients with thymoma: location in the anterior mediastinum; tumor encapsulation; a distinct fat plane between the tumor and vital organs; the existence of residual, normal-appearing thymic tissue; no mass compression effect; and unilateral tumor predominance, particularly for tumors $\geq 3 \mathrm{~cm}$. However, despite that most cases in our series were diagnosed clinically as Masaoka stage I, 49 patients (62\%) were Masaoka stage II after histologic evaluation. A similar finding was reported by Takeo and colleagues, ${ }^{19}$ with $57 \%$ of their patients as Masaoka stage II and stage III after an initial clinical diagnosis of stage I, whereas in a report by Quintanilla-Martinez and associates, ${ }^{24} 28.5 \%$ of the tumors reported to be encapsulated by the surgeon showed microscopic evidence of capsular invasion. No relationship was found between the size of the tumor and Masaoka stage in our series; therefore, a greater size did not predict an increased stage. Our surgical results were good with no mortality, low morbidity, and short hospital stays. The operative time was comparable with other series of thoracoscopic thymoma resection reported in Table 1, but was slightly higher compared with thymectomy for nonthymomatous MG. ${ }^{12,13}$ The learning curve for the no-touch technique and the more complex and accurate dissection required in these cases could explain these results. The open conversion rate was also low $(1.3 \%)$ and comparable with that reported in the literature (range, $0 \%-7.7 \%$ ). With regard to oncologic 
outcome, a recurrence rate of $0 \%$ to $4.5 \%$ has been reported in a thoracoscopic series (Table 1) with variable follow-up (14.5-65 months). Our data agree with the $1.3 \%$ recurrence rate; the single relapse observed was in a type-C thymic tumor, the histology of which was defined only after surgery. No clear evidence of a violation of oncologic principles during the surgical procedure was recorded in this patient. Despite adjuvant treatment, the patient developed a diffuse intrathoracic recurrence, surviving 52 months after the operation. Cheng and coworkers ${ }^{25}$ and Pennathur and colleagues ${ }^{18}$ compared VATS and transsternal approaches for thymoma in a small series and reported no significant difference in recurrence rate or overall survival between the 2 groups. Although encouraging, these results cannot be considered definitive, because the indolent nature of thymoma requires longer follow-up data, such as 10 years, to determine the oncologic outcome of thoracoscopic thymectomy.

The current study had some limitations - in particular, its nonrandomized, retrospective, and multi-institutional design. In addition, although the follow-up was longer than that of most previous studies, it was still inadequate to allow a definitive conclusion on oncologic outcome. In summary, robotic-assisted thoracoscopic thymectomy for early-stage thymomas can be performed safely and efficiently from a technical point of view. In addition to the advantages of the minimally invasive approach (short hospital length of stay, excellent cosmetics, and low morbidity), the increased visualization and instrument dexterity afforded by robotic technology provides further benefit over conventional thoracoscopy. Our data on a relatively large number of patients are encouraging; however, thymoma resection with conventional or robot-assisted thoracoscopy remains under evaluation, because long-term oncologic results are lacking.

\section{References}

1. Regnard JF, Magdeleinat P, Dromer C, Dulmet E, de Montpreville V, Levi JF, et al. Prognostic factors and long-term results after thymoma resection: a series of 307 patients. J Thorac Cardiovasc Surg. 1996;112:376-84.

2. Roviaro G, Varoli F, Nucca O, Vergiani C, Maciocco M. Videothoracoscopic approach for primary mediastinal pathology. Chest. 2000;117:1179-83.

3. Yim AP. Video-assisted thoracoscopic resection of anterior mediastinal masses. Int Surg. 1996;81:350-3.

4. Rea F, Marulli G, Girardi R, Bortolotti L, Favaretto A, Galligioni A, et al. Longterm survival and prognostic factors in thymic epithelial tumours. Eur J Cardiothorac Surg. 2004;26:412-8.

5. Toker A, Erus S, Ozkan B, Ziyade S, Tanju S. Does a relationship exist between the number of thoracoscopic thymectomies performed and the learning curve for thoracoscopic resection of thymoma in patients with myasthenia gravis? Interact Cardiovasc Thorac Surg. 2011;12:152-5.

6. Bodner J, Wykypiel H, Greiner A, Kirchmayr W, Freund MC, Margreiter R, et al. Early experience with robot-assisted surgery for mediastinal masses. Ann Thorac Surg. 2004;78:259-66.

7. Savitt MA, Gao G, Furnary AP, Swanson J, Gately HL, Handy JR. Application of robotic assisted techniques to the surgical evaluation and treatment of the anterior mediastinum. Ann Thorac Surg. 2005;79:450-5.

8. Masaoka A, Monden Y, Nakahara K, Tanioka T. Follow-up study of thymomas with special reference to their clinical stages. Cancer. 1981;48:2485-92.

9. Rosai J, Sobin LH. Histological typing of tumours of the thymus. In: World Health Organization international histological classification of tumours. 2nd ed. New York: Springer; 1999:5-23.
10. Jaretzki A, Barohn RJ, Ernstoff RM, Kaminski HJ, Keesey JC, Penn AS, et al. Myasthenia gravis: recommendations for clinical research standards. Ann Thorac Surg. 2000;70:327-34.

11. Cheng YJ, Hsu JS, Kao EL. Characteristics of thymoma successfully resected by videothoracoscopic surgery. Surg Today. 2007;37:192-6.

12. Rea F, Marulli G, Bortolotti L, Feltracco P, Zuin A, Sartori F. Experience with the "da Vinci" robotic system for thymectomy in patients with myasthenia gravis: report of 33 cases. Ann Thorac Surg. 2006;81:455-9.

13. Rückert JC, Ismail M, Swierzy M. Thoracoscopic thymectomy with the da Vinci robotic system for myasthenia gravis. Ann N Y Acad Sci. 2008;1132:329-35.

14. Toker A, Sonett J, Zielinski M, Rea F, Tomulescu V, Detterbeck FC. Standard terms, definitions, and policies for minimally invasive resection of thymoma. J Thorac Oncol. 2011;6:S1739-42.

15. Davenport E, Malthaner RA. The role of surgery in the management of thymoma: a systematic review. Ann Thorac Surg. 2008;86:673-84.

16. Odaka M, Akiba T, Yabe M, Hiramatsu M, Matsudaira H, Hirano J, et al. Unilateral thoracoscopic subtotal thymectomy for the treatment of stage 1 and 2 thymoma. Eur J Cardiothorac Surg. 2010;37:824-6.

17. Agasthian T, Lin SJ. Clinical outcome of video-assisted thymectomy for myasthenia gravis and thymoma. Asian Cardiovasc Thorac Ann. 2010;18:234-9.

18. Pennathur A, Qureshi I, Schuchert MJ, Dhupar R, Ferson PF, Gooding WE, et al. Comparison of surgical techniques for early-stage thymoma: feasibility of minimally invasive thymectomy and comparison with open resection. $J$ Thorac Cardiovasc Surg. 2011;141:694-701.

19. Takeo S, Tsukamoto S, Kawano D, Katsura M. Outcome of an original videoassisted thoracoscopic extended thymectomy for thymoma. Ann Thorac Surg. 2011;92:2000-5.

20. Mussi A, Fanucchi O, Davini F, Lucchi M, Picchi A, Ambrogi MC, et al. Robotic extended thymectomy for early-stage thymomas. Eur J Cardiothorac Surg. 2012; 41:43-7.

21. Rückert JC, Czyzewski D, Pest S, Müller JM. Radicality of thoracoscopic thymectomy: an anatomical study. Eur J Cardiothorac Surg. 2000;18:735-6.

22. Jaretzki A 3rd. Thymectomy for myasthenia gravis: analysis of controversies: patient management. Neurologist. 2003;9:77-92.

23. Sakamaki Y, Kido T, Yasukawa M. Alternative choices of total and partial thymectomy in video-assisted resection of noninvasive thymomas. Surg Endosc. 2008;22:1272-7.

24. Quintanilla-Martinez L, Wilkins EW Jr, Ferry JA, Harris NL. Thymoma-morphologic subclassification correlates with invasiveness and immunohistologic features: a study of 122 cases. Hum Pathol. 1993;24:958-69.

25. Cheng YJ, Kao EL, Chou SH. Videothoracoscopic resection of stage II thymoma: prospective comparison of the results between thoracoscopy and open methods. Chest. 2005;128:3010-2.

\section{Discussion}

Dr Cameron D. Wright (Boston, Mass). I would like to thank Dr Marulli and his colleagues for a very well-presented series with outstanding results. I appreciate receiving the manuscript for my timely review.

Dr Marulli and his colleagues have described an early analysis of a large series of patients with early-stage thymoma treated with robotic thymectomy with excellent perioperative results from 4 large centers. The operating room times were quite reasonable, complications low and reasonable and not really related to the approach, the median length of stay was short at 3 days, and the recurrence rate observed was quite low. The one caveat of the series is that the median follow-up is relatively short at 3.5 years. There's no question that there was very careful and prudent case selection, and prudent and careful intraoperative operating room judgment exercised, with a no-touch and en bloc technique as well as with total thymectomy. An important caveat is that most recurrences of encapsulated early-stage thymomas occur between 5 years and 10 years in all the series that have been reported to date. Obviously, your follow-up is short and, as you appropriately say, your conclusions are tentative at this point; however, I expect the results 
in the end to be equivalent to the open approach, and I look forward to your publication in another 5 to 10 years describing those results with your excellent judgment and excellent technical skills.

I have just a couple of comments. Just to confirm, because it wasn't described in your paper, were, indeed, all resections an $\mathrm{R} 0$ resection?

Dr Marulli. In our opinion, the resections were all R0 resections. All the specimens were evaluated macroscopically and microscopically after resection.

Dr Wright. You didn't describe in your manuscript the method of follow-up, and obviously clinical follow-up by calling their physician, a telephone call to the patient, or a chest radiograph are all inadequate. The only follow-up that is really adequate for patients with thymoma is periodic computed tomography (CT) scans to look at the mediastinum and the pleural space. Did all of your patients have follow-up CT scans, what was the follow-up protocol, and were there any patients lost to follow-up or not put on a CT periodic surveillance protocol?

Dr Marulli. Obviously, these patients were carefully followed up. Usually, our protocol is to do a clinical in-hospital evaluation of the patient at least every 6 months during the first 3 years, with a CT scan at least every year. Moreover, regarding the patients with MG, all these patients are followed up by a team of neurologists who have a close connection with us, and then in case of variation of clinical status, like a worsening of MG, they are referred to us to evaluate the possible presence of a recurrence. But in all patients, we do a CT scan at least each year.

Dr Joseph B. Shrager (Stanford, Calif). So all of these patients had a CT scan?

Dr Marulli. Yes.

Dr Shrager. Within a year of the study data collection?

Dr Marulli. Yes. The last follow-up was last March.

Dr Wright. The last query is the patient who had a diffuse intrathoracic recurrence. It makes me concerned that there was a capsular violation. I realize it was a type $\mathrm{C}$ patient, but in looking back, were there reasons why that patient would recur diffusely intrathoracically so early?

Dr Marulli. This patient was in the group at Innsbruck and was not a stage I but was a stage IIB patient. We asked to reevaluate the histology of this patient, but I have no reply at this moment. It is possible that the capsule was breached. The patient did adjuvant radiotherapy, but he relapsed intrathoracically with both pleural and intrapulmonary metastases. I don't know if the patient is really type $\mathrm{C}$, but we are evaluating that.

Dr Wright. Thank you very much.

Dr Robert J. Cerfolio (Birmingham, Ala). Excellent presentation. I have some questions about the technical details. We have a paper coming out in the Journal of Thoracic and Cardiovascular Surgery in a month or 2 months, I think, about 160 robotic mediastinal tumors, and a lot of them are thymectomies. I teach a course on this issue and I tell everybody that you should go from the right side, and the reason I prefer the right, and I don't know if you can go back to that picture of yours where you have the one thing just under the left breast, is you're very close to the heart, and in the United States we know of at least 4 patients who had attempted robotic thymectomies in inexperienced hands from the left side and the heart was lacerated, the pericardium was injured, and a few of these patients did not do well. So I feel strongly that we, especially in the beginning, should be going from the right side and not the left. However, I do agree that you can see the right phrenic nerve better from the left chest.

So it would be great if you could educate us on how you're doing it so safely from the left and maybe you can instill your experience and wisdom to us so when we teach inexperienced teams how to do it, we can do it safer from the left. You make the middle incision first, correct? Then put the camera in, insufflate carbon dioxide, you do that first. What port do you put in second? Do you put in the upper axillary port second and then push the heart down as you put in the third and final lower port? What do you do if the heart is big and it's in the way? Teach us some technical tricks.

Dr Marulli. Most patients were operated by the left-sided approach. Only 5 patients had a bilateral approach. We don't feel that it's difficult to see the right phrenic nerve, because if you open the pleura on the-

Dr Cerfolio. No, no. I understand you can see the right phrenic nerve very easily from the left and there is often a tongue of thymus near the aortopulmonary window that is much easier to get out from the left rather than the right. That's not my question. I agree that is the advantage to being on the left. That's not what I'm asking. What I' $m$ asking is, there have been injuries to the heart being on the left side, and as someone who is training others on this, and we will be submitting a manuscript on this, even with a left-sided thymoma, we are going on the right. I'm asking you to tell us how you're avoiding the heart with the sequence of your trocar placement and other technical details to make it safe in inexperienced hands. I want you to educate us on your technique because it seems to be very safe in your hands and not in ours or others. That's the question.

Dr Marulli. We first insert the trocar for the camera port following the lateral aspect of the chest wall to avoid the heart, then we insufflate carbon dioxide, and usually this gives us enough space to place the parasternal trocar without risks of heart injury. Sometimes, for more safety, we use the port at the spot on the third intercostal space going over the heart and taking it down with a cadiere or spatula. By this way, I think it's not dangerous.

Dr Cerfolio. So you're turning your carbon dioxide up, you're putting a spatula to hold the heart down, and then putting that port in last, using a needle to find it. I've tried all those things. I still think we should be on the right, at least to start. I think it's safer on the right.

Dr Wright. Let me just jump up and defend the poor author here. He has an outstanding series of 80 patients with no heart injuries, Cerf. He's doing it the way he likes to do it. We all know that there's a controversy of right versus left, and there's pros and cons to each approach, but I would say that his approach is certainly valid.

Dr Cerfolio. I agree. I didn't say it wasn't. Maybe you didn't hear me, Cam.

Dr Shrager. He said, explain how you can best do it safely.

Dr Cerfolio. I am happy to have someone go back to read what I just said. I never said their technique was not safe. We have a unique opportunity for a world-class group to educate us in this room right now. What I said is, I think it's safer to be on the right for training or to start, and I stand by what I say.

Dr Jens C. Rueckert (Berlin, Germany). I would like to add a comment. With 280 thymectomies, we have had no difficulties 
ever with the left-sided approach. The discussion here is, again, well, I'll take it home because then I see it's right to publish on that question alone. I prepared the manuscript, including all the arguments I heard during the last 15 years, very interesting arguments. I heard that the right-handed surgeon could be better coming from the right side. Maybe. But the radicality is the most important question, and the placement of the trocars should be learned, of course, with insufflation of carbon dioxide in the older or bigger patient, this is correct, but radicality is better from the left side. We include always, even in patients without thymoma, a complete resection, en bloc resection, of the tissue in the aortopulmonary window. This is clearly better from the left side. And the angulation of the typical anatomic site, the anatomic landmark of the innominate, it's better entering the neck from the left side. There is much more to be said. If we discuss the right side for that approach, don't leave the room without the message: the left side.

Dr Alper Toker (Istanbul, Turkey). I would like to know what your attitude is toward lymph node dissection, especially in type $\mathrm{C}$ patients.
Dr Marulli. Obviously, in this case, the histology was known only after the resection. So this patient was a surprise for us. In this case, only adjuvant radiotherapy was considered for this patient. But intraoperatively, the patient had thymoma, apparently encapsulated thymoma, so it was unknown. Usually for stage I and stage II there is no need for lymphadenectomy.

Dr Shrager. Is there a size limit at which you would not approach a thymoma robotically? And for the audience: Is anybody trying to do tumors that might be involving the great vessels robotically?

Dr Marulli. The best indication is less than $3 \mathrm{~cm}$. For robotic, probably less than $5 \mathrm{~cm}$.

Dr Shrager. But your mean size here was $3 \mathrm{~cm}$.

Dr Marulli. Yes. We had 14 patients who had a dimension $>5$ $\mathrm{cm}$. Only 1 was $12 \mathrm{~cm}$. Most of the patients were $<8 \mathrm{~cm}$. If the tumor is unilateral in the lower part of the thymus, probably resection can be safe with easy access to the contralateral mediastinum and the neck. In these cases, you can do also a tumor $>5 \mathrm{~cm}$. A good indication is less than $5 \mathrm{~cm}$. 